\title{
Electrode Degradation in PEMFs as Studied in Model Systems and PEMFC Testing
}

\author{
F.A. de Bruijn \\ V.A.T Dam \\ G.J.M. Janssen \\ R.C. Makkus
}

Published in the ECS Transacions, 25 (1) 1835-1847 (2009) 



\title{
Electrode Degradation in PEMFCs as Studied in Model Systems and PEMFC Testing
}

\author{
Frank A. de Bruijn ${ }^{1,2}$, Van Anh T. Dam ${ }^{2}$, Gaby J. M. Janssen ${ }^{1}$, Robert C. Makkus ${ }^{1}$ \\ ${ }^{1}$. Energy research Centre of the Netherlands \\ PO Box 1, 1755 ZG Petten, Netherlands \\ 2. University of Technology Eindhoven, PO Box 513, 5600 MB Eindhoven
}

In order to meet the durability criteria for PEM Fuel Cells, needed for their successful application in transport, more insight is needed into which conditions have a detrimental effect on the long term performance, and which materials improvements are needed to make PEMFC better suited to withstand some of these conditions that are unavoidable in practice.

To obtain such insights ex situ model studies under well-defined conditions as well as in situ accelerated stress tests under more realistic conditions have been combined. MEAs using state of the art materials have been subjected to 57 freeze/thaw cycles or 30,000 voltage cycles. Both conditions lead to a decrease in electrochemical surface area; in addition, voltage cycling leads to significant oxygen transport losses. Model studies in perchloric acid revealed that both platinum and carbon corrode when exposed to high potentials at high temperatures. Decreasing temperature leads to a significant decrease in the corrosion rates.

\section{Introduction}

For its successful application in transport, PEM fuel cells need to be able to withstand conditions that fall outside their ideal operating window. This ideal operating window was identified as (1): operation at constant voltage at a relative humidity close to $100 \%$, at a temperature of $75^{\circ} \mathrm{C}$ at maximum and using an optimal stack and flow design. Voltage decay rates under these conditions can be as low as 1-2 $\mu \mathrm{V} \cdot \mathrm{hr}^{-1}$. Conditions that fall outside the ideal operating window are, amongst others: freeze/thaw cycles and voltage cycles, especially when including periods at open circuit. Such conditions can lead to voltage decay rates that are orders of magnitude higher than when operated under ideal conditions, and even to complete MEA failure.

In order to keep the voltage decay rate to an acceptable level and prevent MEA failure, more robust materials need to be developed that can withstand the off-spec conditions. As long as such materials are not available, damage might be controlled by preventing the most stressing conditions. In either case, more insight is needed on which exact conditions lead to an increase in voltage decay or MEA failure, and to which materials changes they lead.

In this paper, tests on MEA's exposed to stressing conditions combined with extensive characterization are described, as well as model studies on platinum, carbon and platinum/carbon electrodes. 


\section{MEA testing in temperature cycles}

\section{Test protocol of temperature cycles}

The effect of Freeze/Thaw (F/T) cycles down to $-20{ }^{\circ} \mathrm{C}$ without removal of water prior to freezing was studied using a $7 \mathrm{~cm}^{2}$ MEA with state of the art materials, Nafion ${ }^{\circledR} 212$, Sigracet $^{\circledR}$ GDL31BC from SGL Carbon and Hispec ${ }^{\circledR} 9100$ catalyst from Alfa Aesar; the catalyst loading was $0.4 \mathrm{mg} / \mathrm{cm}^{2}$ for cathode and anode. A similar MEA was used for start/stop (S/S) cycles down to $5^{\circ} \mathrm{C}$. Each cycle consisted of:

1. Operation at $65{ }^{\circ} \mathrm{C}$ with almost fully humidified gases, a stoichiometry of 2 for both air and hydrogen at a current density of $400 \mathrm{~mA} / \mathrm{cm}^{2}$,

2. Switching over to OCV immediately followed by a closing of the gas supply and a forced cooling, either down to $5^{\circ} \mathrm{C}$ or $-20{ }^{\circ} \mathrm{C}$;

3. A 2 hour (at day time), or 15 hour (at night time) period at the lowest temperature;

4. Heating up to $65^{\circ} \mathrm{C}$. Upon reaching $65^{\circ} \mathrm{C}$ the gases are switch on and the current density is set to $400 \mathrm{~mA} / \mathrm{cm}^{2}$.

At regular intervals extensive electrochemical characterization was carried out, which included $j-V$ recordings as well as cyclic voltammograms and electrochemical impedance spectroscopy to obtain the ohmic resistance of the cell and the protonic resistance of the cathode catalyst layer. The protonic resistance of the catalyst layer $R_{p}$ was derived from the high frequency (h.f) region of the impedance spectrum $Z^{h f}(\omega)$ according to (2):

$$
Z^{h f}(\omega)=R+\sqrt{\frac{R_{p}}{C_{d l}}} \omega^{-1 / 2} \frac{\sqrt{2}}{2}(1-i)
$$

Here $R$ is the intercept with the real axis at high frequency to be identified with the ohmic resistance. Equation 2 implies that the h.f. part of the impedance Nyquist plot has. a region where the Nyquist plot is a straight line with a $45^{\circ}$ angle to the real axis. This part of the spectrum usually corresponds to frequencies between 1 and $10 \mathrm{kHz}$. At higher frequency the impedance is dominated by inductance effects and at lower frequency by the charge transfer reaction Points belonging to the $45^{\circ}$ region were selected and a fit of equation 9 was made. The double layer capacitance $C_{d l}$ was obtained from cyclic voltammograms

\section{$\underline{\text { Results of temperature cycles }}$}

The F/T MEA was subjected to 57 cycles, whereas the other MEA was subjected to $58 \mathrm{start} / \mathrm{stop}$ cycles. The polarization curve of a fresh MEA is compared to the $j-V$ characteristics of the MEA and the S/S MEA in Figure 1. Due to the temperature cycling 
and aging the performance is decreased, but comparable for both MEAs.

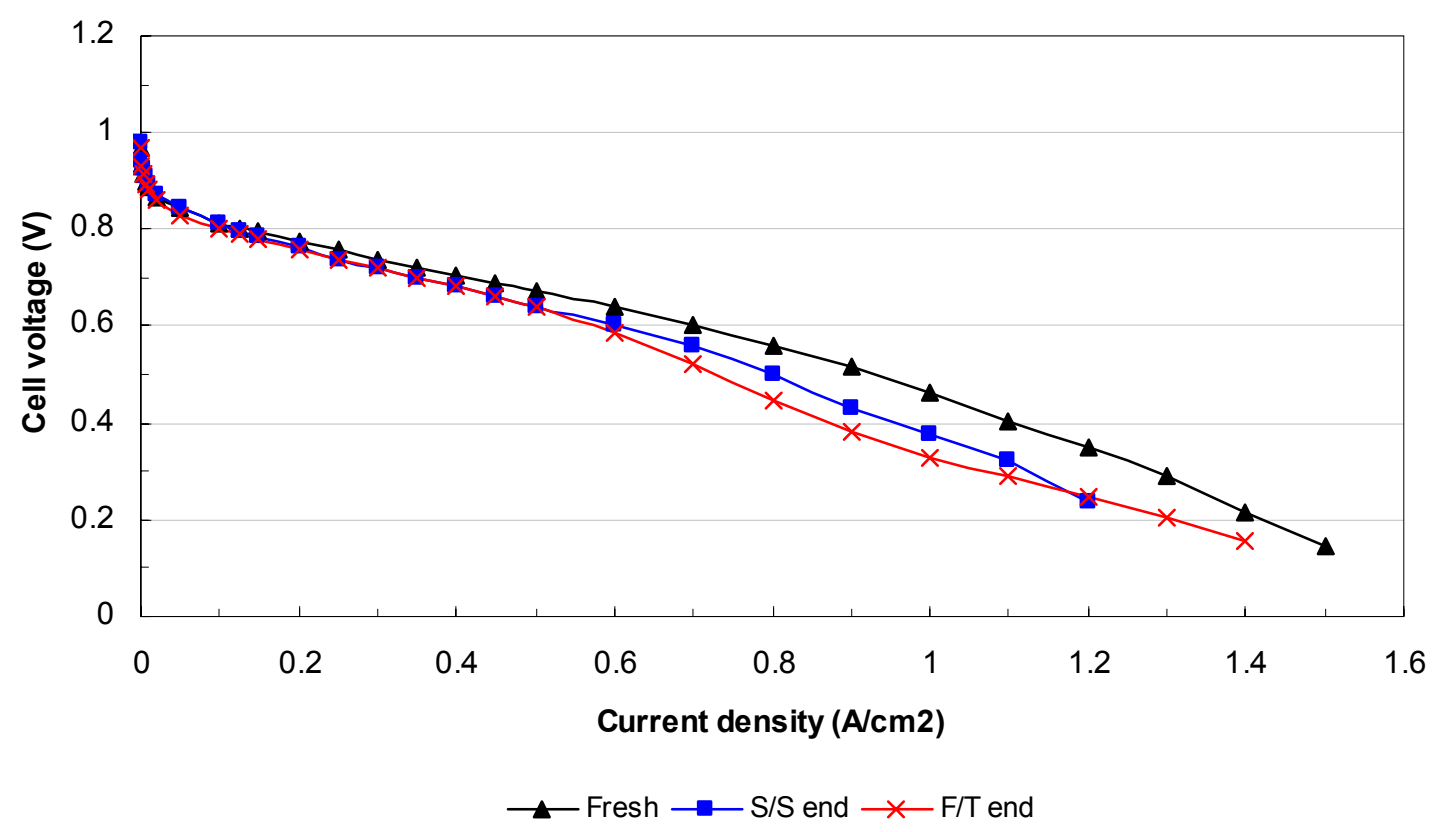

Figure 1. The polarization characteristics of a fresh MEA and the F/T and S/S MEA after 57 and 58 cycles respectively. Cycling and aging result in a lower performance. Cell temperature was $65{ }^{\circ} \mathrm{C}$; atmospheric pressure; $\lambda_{\mathrm{H} 2}=1.5, \lambda_{\text {air }}=2$; both dew points were approximately $65^{\circ} \mathrm{C}$.

During the testing period the gas cross-over remained at the same level $1.1-1.2$ $\mathrm{mA} / \mathrm{cm}^{2}$ for both MEAs. Also the ohmic resistance was not affected, in contrast to the observation by Cho et al. $(3,4)$. Figure 2 shows the ohmic resistance of the F/T MEA. A small effect of the current density can be observed, a higher current density results in a lower ohmic resistance due to the increased amount of water present in the catalytic layer. The fact that the ohmic resistance does not increase as a result of the F/T cycles indicates that there is no delaminating of the different MEA layers. 


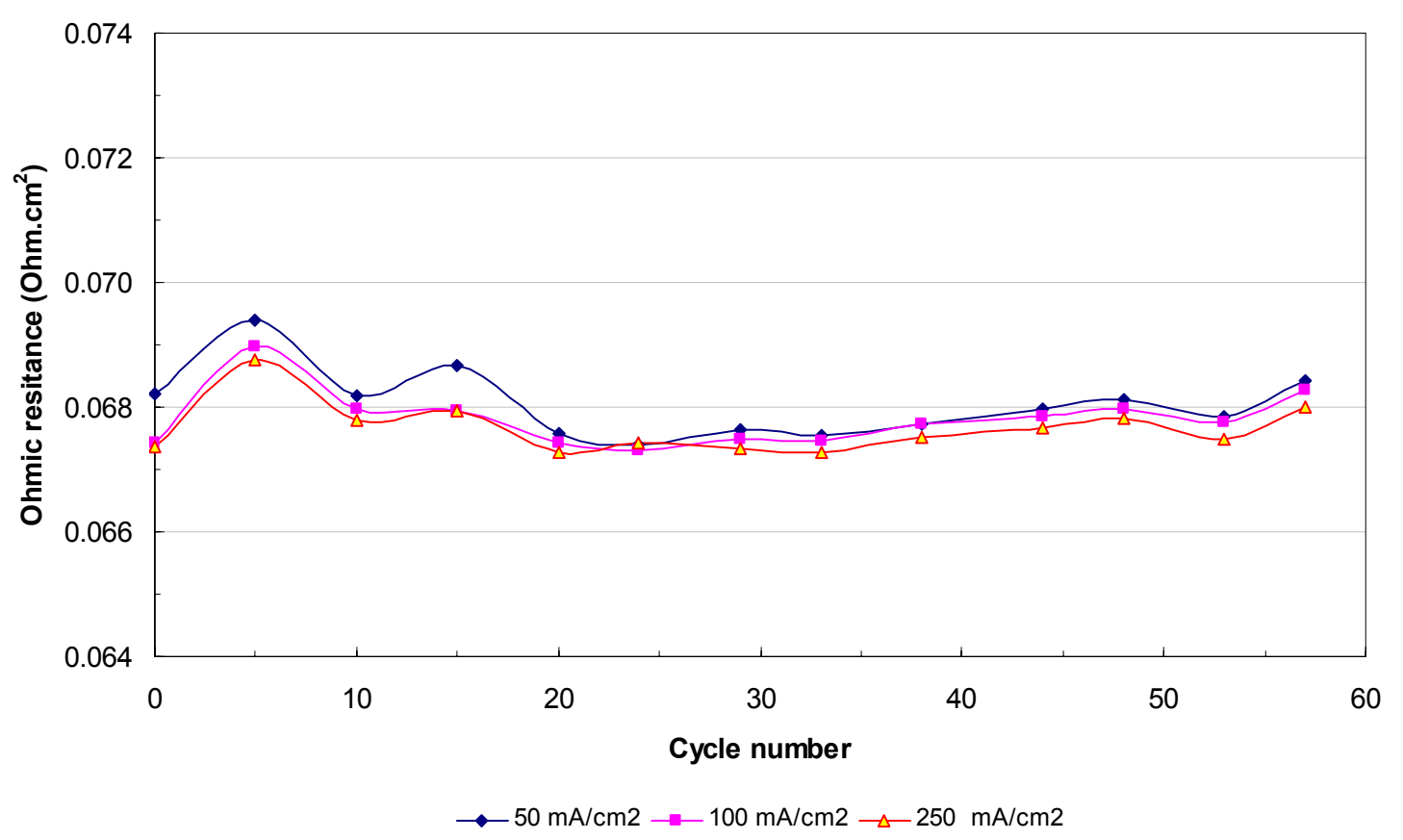

Figure 2. The ohmic resistance of an MEA exposed to 57 Freeze/Thaw cycles determined at different current densities. Cell temperature was $65{ }^{\circ} \mathrm{C}$; atmospheric pressure; $\lambda_{\mathrm{H} 2}=1.5, \lambda_{\text {air }}=2$; both dew points were approximately $65{ }^{\circ} \mathrm{C}$.

For both MEAs the electrochemically active surface area (ECSA) decreased due to either Pt particle growth or Pt dissolution, by $37 \%$ for the F/T MEA and by $48 \%$ for the $\mathrm{S} / \mathrm{S}$ MEA. Consequently the mass activity was reduced after an initial increase. The surface area loss corresponds to a voltage loss of 13 to $18 \mathrm{mV}$, which is confirmed by Tafel plots. The polarization resistance as determined under oxygen using EIS has increased in time; the relative increase was $18 \%$ at $50 \mathrm{~mA} / \mathrm{cm}^{2}$ increasing to 21 , and $23 \%$ for 100 , and $250 \mathrm{~mA} / \mathrm{cm}^{2}$ respectively for both MEAs. This increase must be related to transport effects. A reduction in kinetics alone would, in the Tafel regime, not result in a higher impedance. Also the fact that the relative increase is largest at high current density points in that direction. In addition the oxygen gain at $j=400 \mathrm{~mA} / \mathrm{cm}^{2}$ has increased about $20 \mathrm{mV}$, which is also indicative of increased transport losses.

A difference was observed for the proton resistance of the cathode catalyst layer; the start/stop cycles did not affect this resistance, whereas it decreased for the F/T MEA to the same value as for the S/S MEA, which is more or less a minimal value (Figure 3). The cause of the difference in initial value is unclear as both MEAs are made according to the same protocol. A decrease in proton resistance can be explained by a rearrangement of the ionomer phase inside the catalyst layer or an increased hydrophilicity. These can be the result of cycling as well as operation time.

In conclusion, the effect of a limited number of $\mathrm{F} / \mathrm{T}$ cycles with water remaining in the cell on the cell performance and MEA characteristics is not larger than of MEAs undergoing S/S cycles. Also Guo et al. (5) and Mukundan (6) have observed that for a low number of $\mathrm{F} / \mathrm{T}$ cycles the performance of an MEA is not affected. 


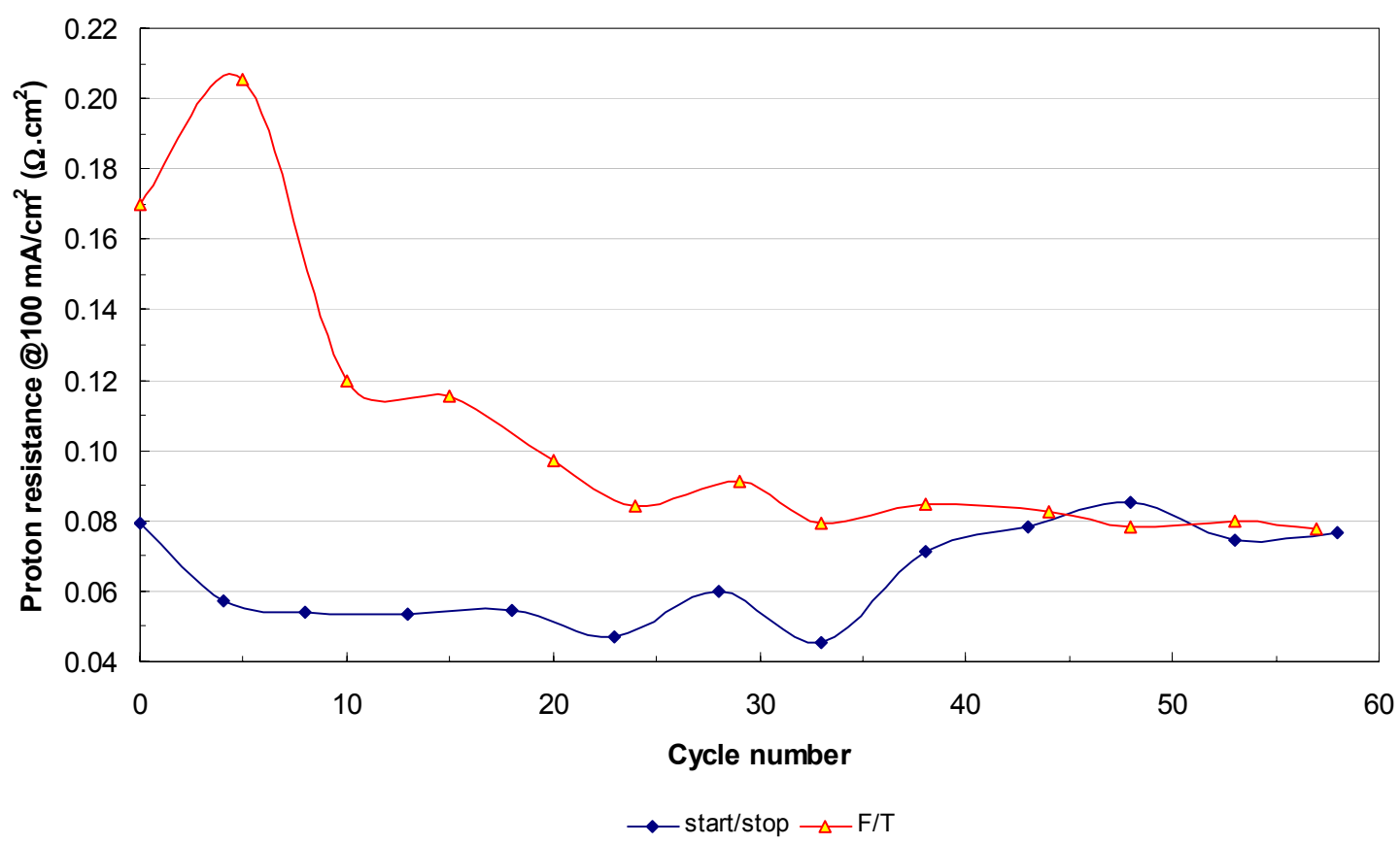

Figure 3. The proton resistance of the cathode catalyst layer for MEAs exposed to start/stop cycles and Freeze/thaw cycles as determined according to equation 1.

\section{MEA testing in voltage cycles}

\section{Test protocol of voltage cycles}

The test protocol used for voltage cycles was a modified version of the DOE protocol for fast potential cycling (7). The total amount of cycles and the potential range in the DOE protocol are considered to be relevant for the target of $5000 \mathrm{~h}$ operating time for automotive applications. In contrast with the DOE protocol the cycling was carried out while operating the cell with $\mathrm{H}_{2} /$ air instead of $\mathrm{H}_{2}$ /nitrogen. Hence, the tests also involved load, and therefore humidity, cycling. This presents also stressing conditions for the membrane and ionomer phase in the electrode (1) whereas the DOE protocol targets solely catalyst degradation.

During the fast voltage cycling membrane-electrode-assemblies (MEAs) were operated with $\mathrm{H}_{2}$ (stoichiometry 1.5) and air (stoichiometry 2) and underwent 30000 square wave cycles between $0.7 \mathrm{~V}$ (IR-corrected) and $0.9 \mathrm{~V}$ (IR-corrected), or OCV when OCV dropped below $0.9 \mathrm{~V}$, with $30 \mathrm{~s}$ hold at each potential. The operating temperature was $80^{\circ} \mathrm{C}$ with the gases at atmospheric pressure and humidified at $80 \% \mathrm{RH}$. Two types of MEAs were considered. MEA1 contained a NRE211CS membrane from DuPont and two identical electrodes consisting of a catalytic layer with $57 \mathrm{wt} \% \mathrm{Pt}$ on high surface area carbon black (Hispec ${ }^{\circledR} 9100$ from Alfa Aesar) and a Nafion ${ }^{\circledR}$ to carbon weight ratio of 0.7 on Sigracet $^{\circledR}$ GDL31BC from SGL Carbon. For MEA2 a thicker membrane, NRE212CS, was used and the Nafion ${ }^{\circledR}$ to carbon weight ratio in the catalyst layer was decreased to 0.55 . In all electrodes the Pt loading was in the order of $0.4 \mathrm{mg} / \mathrm{cm}^{2}$. The preparation of the MEAs has been described elsewhere (2). 
After $0,1000,5000,10000$ and 30000 cycles electrochemical characterization was carried out which comprised recording $j-V$ characteristics in $\mathrm{H}_{2}$ /air as well as in $\mathrm{H}_{2} / \mathrm{O}_{2}$, cyclic voltammograms of the cathode, electrochemical measurement of the hydrogen cross-over, and an impedance spectrum of the MEA while operating under $\mathrm{H}_{2} / \mathrm{O}_{2}$ at 50 $\mathrm{mA} / \mathrm{cm}^{2}$. The electrochemical characterization was described in more detail in a previous paper (2). Between the full characterization steps additional cyclic voltammograms were recorded according to the DOE scheme (7). Post test, the cross sections of the MEAs were examined by SEM. The cathode catalyst layer was scraped off the GDL with a razor blade and the resulting powder was analyzed by TEM.

\section{$\underline{\text { Results of voltage cycles }}$}

The extended electrochemical characterization enables a study of the time evolution of the components that constitute the cell voltage losses, i.e. kinetic, ohmic, and transport losses. In this analysis, it is assumed that anode kinetic losses are negligible. From the cyclic voltammograms the ECSA of the cathode was determined. Figure 4 shows that in both MEAs the ECSA decreased substantially. Post test TEM analysis indicated that Pt particle growth had occurred from average 3.0 to $5.8 \mathrm{~nm}$ but also some Pt was found in the membrane. The loss of ECSA is reflected in the loss of mass activity of the MEAs. The values for mass activity are derived from the $j-V$ characteristics measured with oxygen and refer to the activity at $0.9 \mathrm{~V}$ (IR-corrected) and 1 bar oxygen pressure. The increase $\triangle \eta_{O R R}$ in kinetic loss according to the reduction of an initial value of the mass activity $i_{m}{ }^{0}$ at 0 cycles to $i_{m}{ }^{n}$ at $n$ cycles is given by:

$$
\Delta \eta_{O R R}=b \log \frac{i_{m}^{0}}{i_{m}^{n}}
$$

In this equation $b$ is the Tafel slope with a value in the order of $67 \mathrm{mV} / \mathrm{dec}$. It should be noted that although the kinetic losses increase with current density and are larger in air than in oxygen, the change in kinetic loss is independent of the current density as well as of the oxidant. Note, that the mass activity values at 30000 cycles could either not be determined or were not very reliable as the value of $0.9 \mathrm{~V}$ (IR-corrected) was above OCV in MEA1 with the thin membrane, or close to it in MEA2. However, the results clearly indicate that the DOE targets of less than $40 \%$ loss of ECSA and less than $60 \%$ loss of mass activity after 30000 cycles are not met by these MEAs.

The drop in OCV results from the reduced mass activity but also from increased hydrogen cross-over due to membrane damage (7). Increased hydrogen cross-over was observed for the NRE211CS membrane, where between 10000 and 30000 values the equivalent cross-over current density increased from the initial value of $3.5 \mathrm{~mA} / \mathrm{cm}^{2}$ to $9.8 \mathrm{~mA} / \mathrm{cm}^{2}$. In the MEA with a thicker NRE212CS membrane the value remained constant at $1.2 \mathrm{~mA} / \mathrm{cm}^{2}$. The ohmic resistance $R$ of the MEAs as measured by impedance spectroscopy showed a small reduction of $0.005 \mathrm{Ohm} . \mathrm{cm}^{2}$ in both MEAs. The change in ohmic loss between cycle 0 and cycle $n$ can be calculated from $\Delta \eta_{\Omega}=j \cdot\left(R^{n}-R^{0}\right)$. The SEM analysis showed a reduction of the NRE211CS membrane thickness by $40 \%$ after cycling, whereas the thickness of NRE212CS membrane seemed unchanged. 

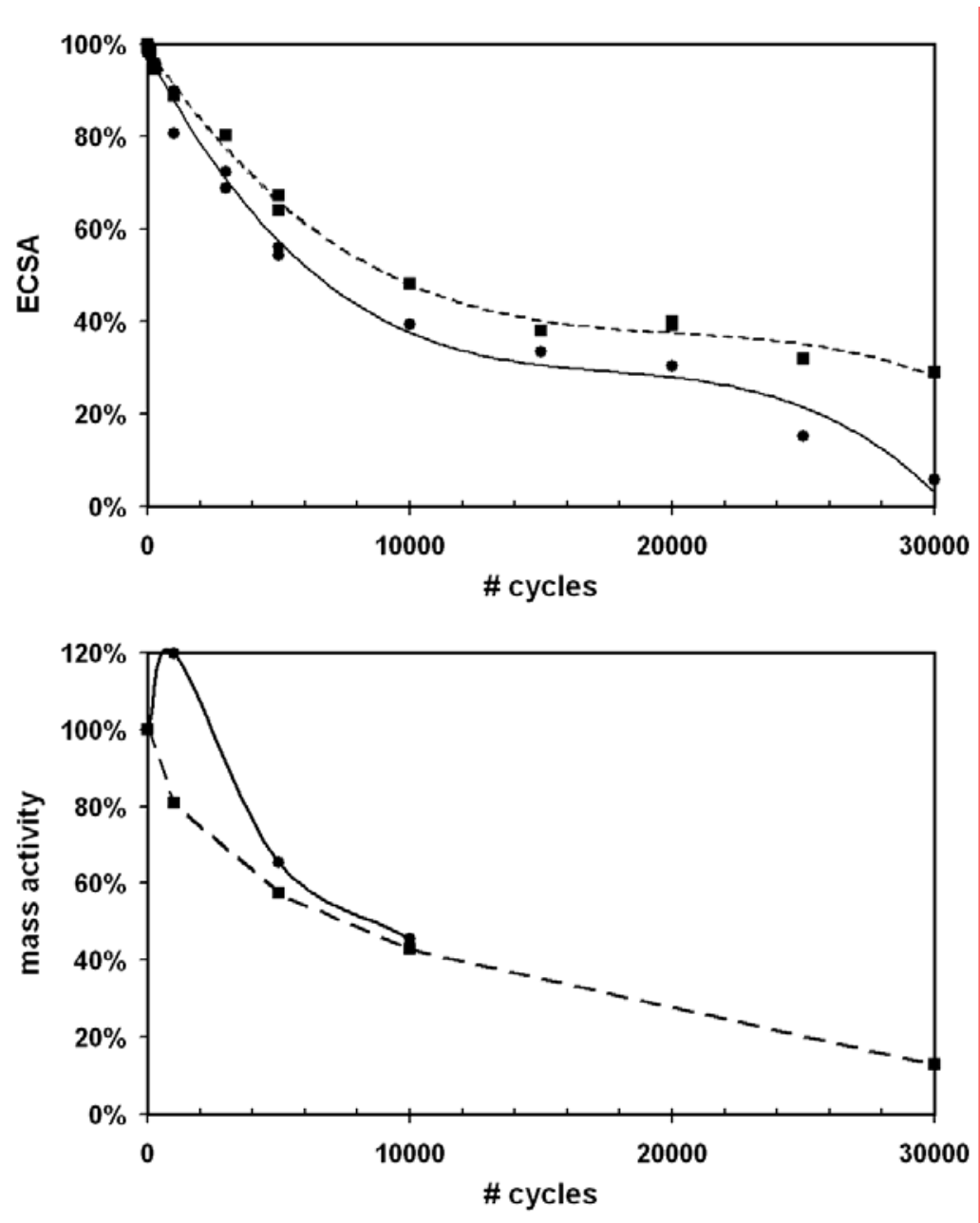

Figure 4. The ECSA (top) and mass activity (bottom) of MEA1 ( $\bullet$, drawn lines) and MEA2 ( $\mathbf{n}$, dashed lines) as a function of the number of cycles. The values were normalized to the initial values. Cell temperature was $80^{\circ} \mathrm{C}$; atmospheric pressure; $\lambda_{\mathrm{H} 2}=$ $1.5, \lambda_{\text {air }}=2$; relative inlet humidity $80 \%$.

Figure 5 shows the dependence on number of cycles of $\Delta \eta_{O R R}$ and $\Delta \eta_{\Omega}$ as well as the total change in cell voltage loss $\Delta \eta_{\text {air }}$ at $j=400 \mathrm{~mA} / \mathrm{cm}^{2}$. As $\Delta \eta_{\Omega}$ can be neglected, the difference between $\Delta \eta_{\text {air }}$ and $\Delta \eta_{O R R}$ must be ascribed to transport losses. As Figure 5 shows, the transport losses are of similar magnitude as the kinetic losses and depend on ionomer content of the catalyst layer. Both limitations in proton transport and oxygen transport contribute to these transport losses. A measure for the proton transport loss is the proton resistance of the catalyst layer as can be obtained from the high-frequency part of the impedance spectrum (2). In MEA1 with high ionomer content the value was constant $\left(0.4 \mathrm{Ohm} . \mathrm{cm}^{2}\right)$ whereas in the MEA2 with low ionomer content the value decreased from 0.8 to $0.4 \mathrm{Ohm} . \mathrm{cm}^{2}$, similarly to what was found in a previous study on, less intensive, load cycling (2). The result is also corroborated by a comparison of $\triangle \eta O R R$ and $\Delta \eta_{O 2}$. The latter term represents the total change in cell voltage loss for a cell operated under oxygen. In this case proton transport will dominate the transport losses. In the case of low ionomer content, the $\Delta \eta_{O 2}$ was smaller than the $\Delta \eta_{O R R}$ in agreement with reduced transport loss. 

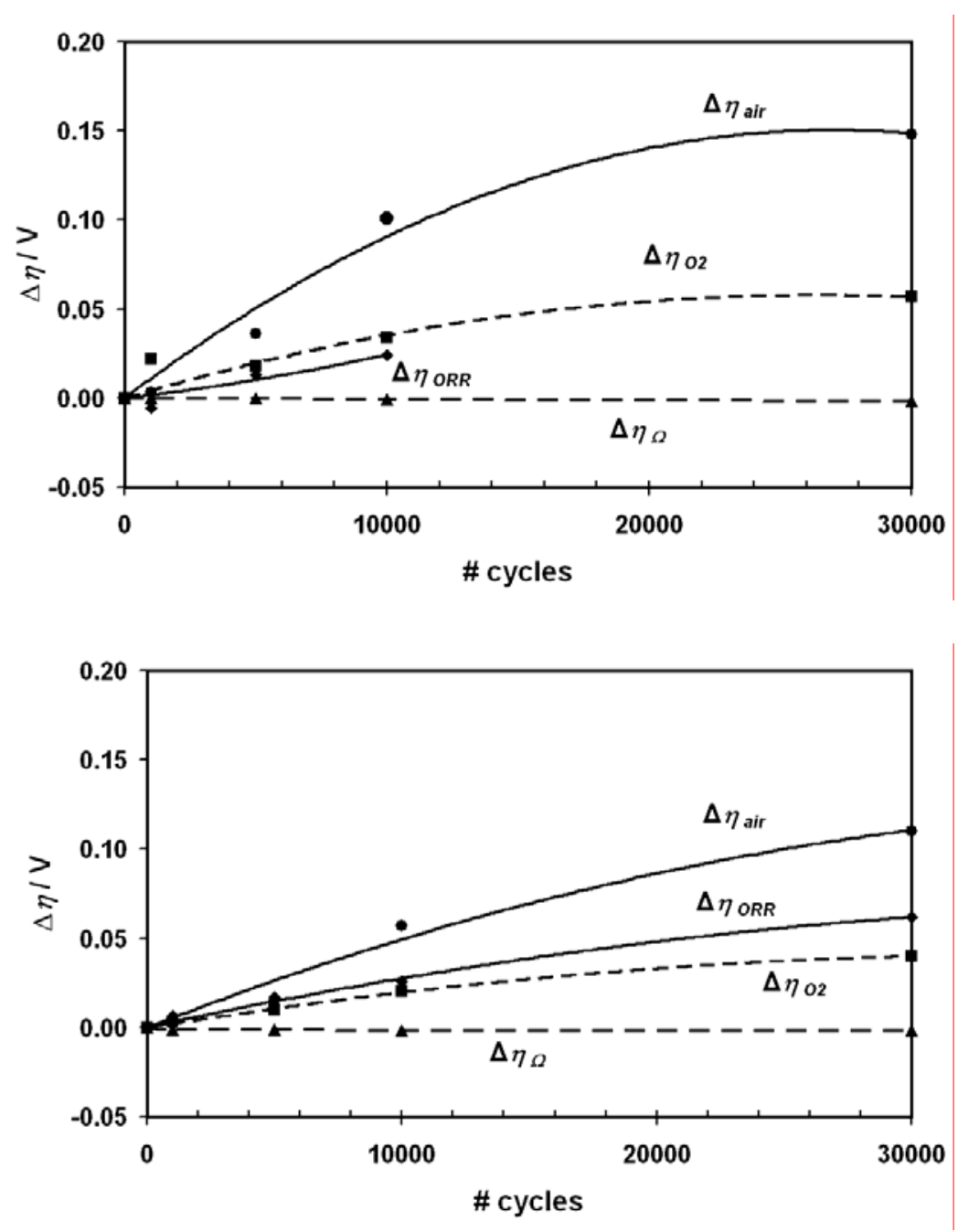

Figure 5. The change in cell voltage loss at $400 \mathrm{~mA} / \mathrm{cm}^{2}$ of MEA1 (top) and MEA2 bottom) undergoing potential cycling, when operated under air $\Delta \eta_{\text {air }}$ under oxygen $\Delta \eta_{O 2}$ as well as the changes in ohmic $\Delta \eta_{\Omega}$ and kinetic $\Delta \eta_{O R R}$ losses. Cell temperature was $80^{\circ} \mathrm{C}$; atmospheric pressure; $\lambda_{\mathrm{H} 2}=1.5, \lambda_{\text {air }}=2, \lambda_{\mathrm{O} 2}=9.5$; relative inlet humidity $80 \%$.

The difference between $\Delta \eta_{O 2}$ and $\Delta \eta_{\text {air }}$ is the change in the so-called oxygen gain. As the proton resistance does not increase, the increased oxygen gain indicates a reduced oxygen permeability of the cathode. Although contributions from GDL and catalyst layer cannot be rigorously separated on the basis the present measurements, it is clear that, like in previous load-on/off cycles, the increase of transport losses depends strongly on the catalyst layer composition (2). Reduced hydrophobicity of this layer may explain both the reduced proton resistance as well as the lower oxygen permeability. 


\section{Model Studies on Platinum, Carbon and Platinum/Carbon electrodes}

Although fuel cell studies on MEA's are the most representative, it is difficult to isolate the degradation mechanisms that can take place simultaneously. A good example of this is the long term performance study by Cleghorn et al (8). During the 26,000 hours of PEMFC operation, in-situ and ex-situ characterization revealed a loss of water removal efficiency, the detoriation of seals, loss of Pt surface area in the cathode, thinning of membrane and an increased hydrogen cross-over. At the same time, such a MEA has experienced a wide range of conditions, such that one can only indirectly link materials changes to operating conditions.

To obtain more insight in the influence of the electrode potential on the stability of carbon and platinum, electrochemical studies were performed under primarily potentiostatic conditions at temperatures relevant for PEM fuel cells operation.

\section{Experimental methods in Model Studies}

By applying a Quartz Crystal Micro Balance, mass changes in the working electrode can be measured with a high accuracy. For platinum, carbon and platinum/carbon catalysts, a gold/titanium quartz crystal substrate was used. For studying the electrochemical stability of platinum thin films, the platinum was deposited on a polished gold/titanium electrode by the reduction of platinum from a $0.02 \mathrm{mM} \mathrm{H}_{2} \mathrm{PtCl}_{6} / 0.02 \mathrm{M} \mathrm{Na}_{2} \mathrm{HPO}_{4}$ solution. In case of carbon and platinum/carbon, an ink containing Vulcan XC72R or Johnson Matthey Hispec $^{\mathrm{TM}} 4000$ platinum/carbon, Nafion and 1,2-propanediol was applied on an unpolished gold/titanium quartz crystal substrate. The stability of platinum, carbon and platinum/carbon was studied by monitoring the mass of these resulting electrode while applying a constant potential in the range of $0.85-1.4 \mathrm{~V}$ at 60 and $80{ }^{\circ} \mathrm{C}$ in $1 \mathrm{M} \mathrm{HClO}_{4}$. The electrode surface was characterized at different intervals by applying cyclic voltammetry. Transmission Electron Microscopy was used on selected samples to determine the platinum particle size distribution. Full experimental details are described in (9) for platinum and (10) for carbon and platinum/carbon.

\section{$\underline{\text { Platinum }}$}

Studying the stability of platinum in platinum thin films allows the direct measurement of platinum dissolution, as no interfering species are present in the system. By studying the mass change of the electrode versus time at various potentials and temperatures, it was found that (8):

- An increase of the electrode mass, linked to the build up of an oxide layer, always precedes the decrease of the electrode mass, linked to the dissolution of this oxide layer

- Even after measuring a substantial loss in electrode mass, a thick oxide layer could be measured by cyclic voltammetry. Figure 6 (left), shows the oxide reduction peak after potentiostatic hold at $1.05,1.15$ and $1.25 \mathrm{~V}$. This reduction peaks are measured after dissolving a substantial part of the platinum thin film. This makes it very likely that platinum is dissolved by the dissolution of its oxide, rather than the direct corrosion of metallic platinum

- The dissolution rate, as calculated from the loss of electrode mass in time, increases exponentially with the electrode potential, but shows a maximum at $80^{\circ} \mathrm{C}$ and $1.15 \mathrm{~V}$, see Figure 6 (right). This is an indication that deep oxides are insoluble and passivate the platinum. 
- temperature has a huge effect on the dissolution rate: at $60{ }^{\circ} \mathrm{C}$, the measured dissolution rate is a factor 1000 lower than at $80^{\circ} \mathrm{C}$, see Figure 6 (right).
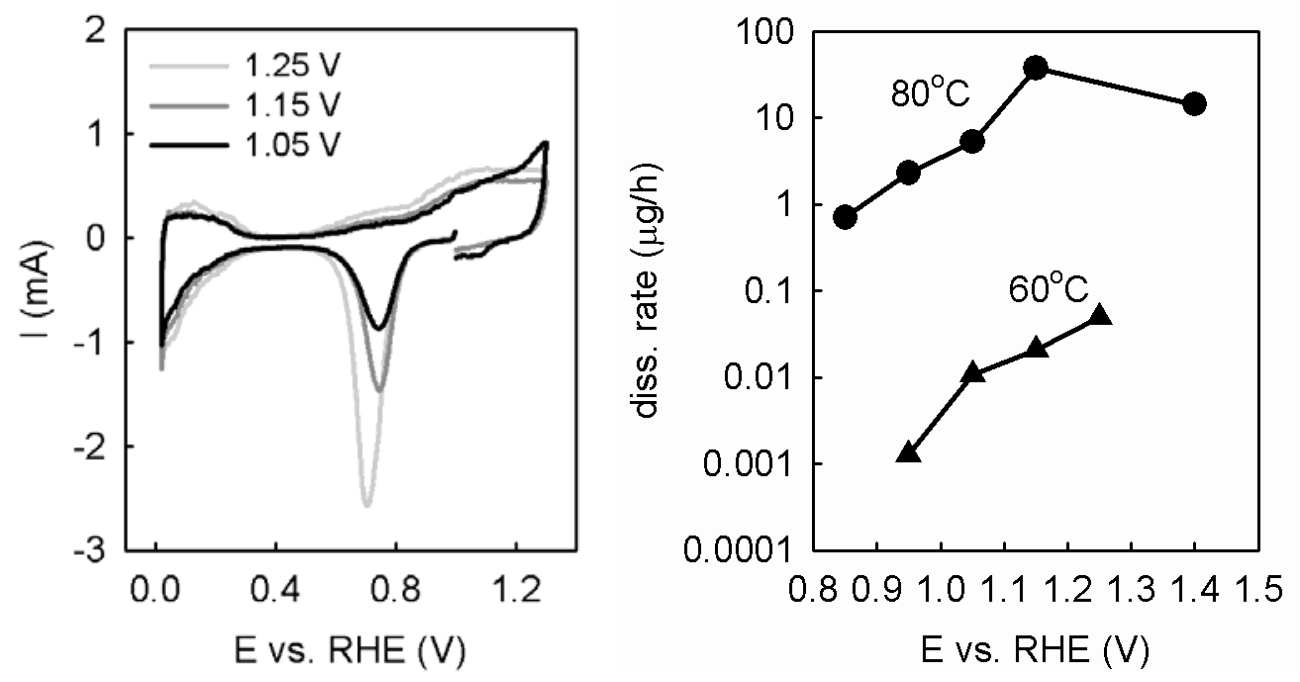

Figure 6. Platinum dissolution from electrodeposited platinum under potentiostatic conditions in $1 \mathrm{M} \mathrm{HClO}_{4}$. Left: platinum oxides still present after significant amount of dissolved platinum, $60{ }^{\circ} \mathrm{C}$. Right: platinum dissolution rate versus potential and temperature, as calculated from electrode mass decrease versus time. Previously published in Journal of Electrochemical Society (9).

\section{Carbon}

The stability of carbon was studied at potentiostatic conditions in the potential range between $0.95 \mathrm{~V}$ and $1.15 \mathrm{~V}$ at $60^{\circ} \mathrm{C}$ and $80^{\circ} \mathrm{C}$. Mass changes as well as measuring the $\mathrm{CO}_{2}$ production by a mass spectrometer revealed that, as in the case of platinum, temperature and potential have a profound influence on carbon stability. Under potentiostatic conditions, at potentials below $1.15 \mathrm{~V}$, no indication of carbon corrosion was found neither at $60{ }^{\circ} \mathrm{C}$ nor at $80{ }^{\circ} \mathrm{C}$ by QCM. Characterization by cyclic voltammetry revealed that the observed mass increase can be linked to the formation of quinone groups. At $1.15 \mathrm{~V}$, the mass increase shows an accelerating pattern, followed by a sudden, sharp decline in mass. On line mass spectrometry revealed $\mathrm{CO}_{2}$ formation at the same conditions during a potential sweep, which indicates that during the mass increase as observed by QCM, $\mathrm{CO}_{2}$ formation is probably already taking place, albeit in very small quantities when compared to that linked to the sharp mass decrease after longer hold times. Decreasing the temperature from $80^{\circ} \mathrm{C}$ to $60{ }^{\circ} \mathrm{C}$ delays the moment of sudden mass decrease from 5 to 25 hours.

These data show that during normal operation, with OCV potentials around 0.9 to $1.0 \mathrm{~V}$ being the highest potentials to which the cathode will be exposed, continuous carbon corrosion is not expected based on these observations. High potentials occurring during start/stop cycles can lead to severe carbon corrosion when the potentials persist for too many hours. 

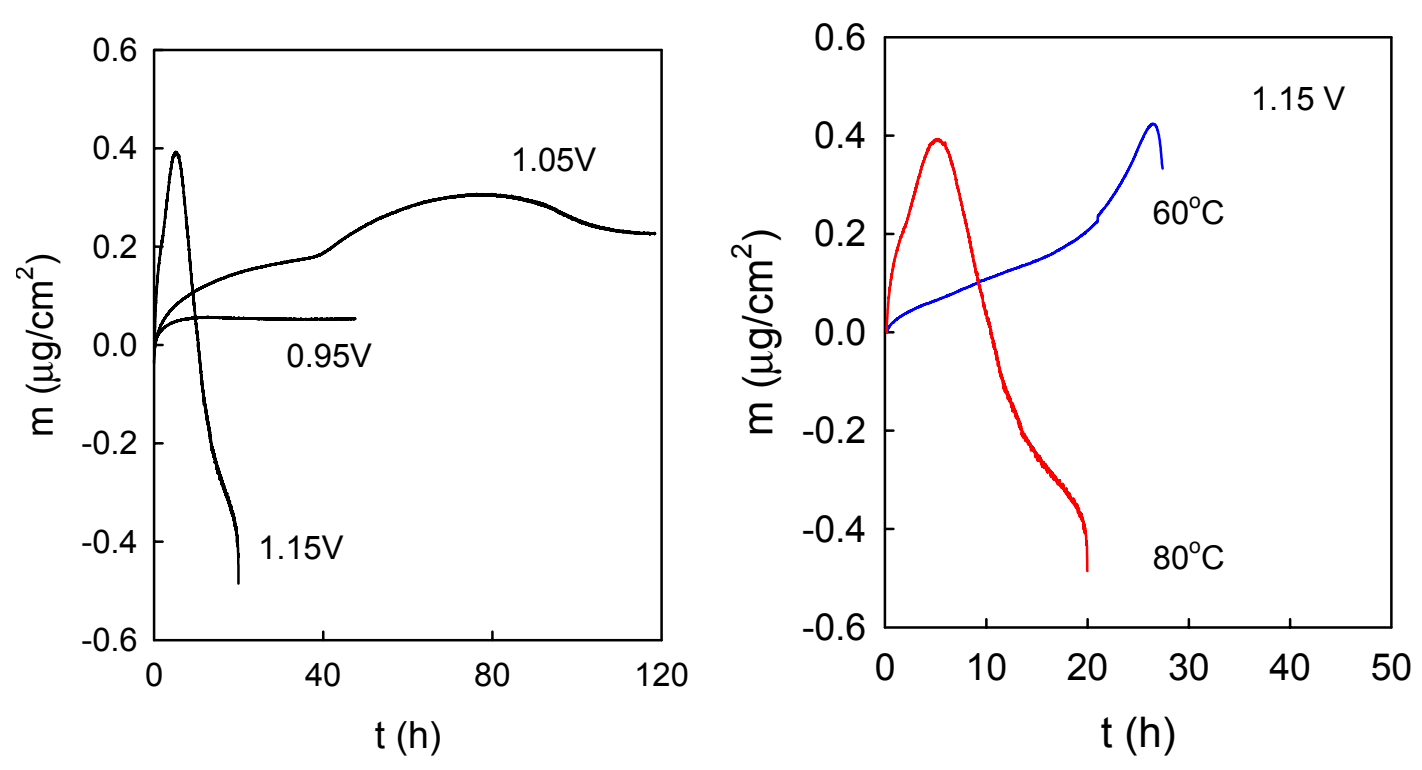

Figure 7. Mass of carbon electrode as measured by QCM under potentiostatic conditions, $1 \mathrm{M} \mathrm{HClO}_{4}$. Left: Influence of potential, at $80^{\circ} \mathrm{C}$. Right: Influence of temperature, $1.15 \mathrm{~V}$. Figure previously published in Fuel Cells (10).

\section{Platinum on Carbon}

Platinum on carbon represents the most complex system, and necessitates the use of various techniques to draw conclusions on the materials changes taking place. Cyclic voltammetry, QCM and TEM were combined before final conclusions could be drawn. Under all conditions studied, a linear decrease of the platinum surface area in time was measured by cyclic voltammetry at different intervals during the potentiostatic hold. Figure 8 (left) shows the decrease in platinum surface area versus time at $1.05 \mathrm{~V}, 80^{\circ} \mathrm{C}$. In 118 hours, more than $80 \%$ of the surface area is lost. A typical observation in the cyclic voltammograms was the shift of the platinum oxide reduction peak to lower potentials after increasing potentiostatic hold times, indicating a decrease in platinum particle size. This indication was confirmed by TEM, Figure 8 (right), which shows a marked decrease in the amount of platinum particles on the support, as well as a decrease of the average particle size from $4 \pm 1.2 \mathrm{~nm}$ to $3 \pm 1.4 \mathrm{~nm}$. These observations lead to the conclusion that the observed decrease in platinum surface area is caused by platinum dissolution, rather than Ostwald ripening under the conditions applied in the model studies.

Over the whole potential and temperature range studied, the observations with respect to the stability of platinum are in line with that on pure platinum:

- Platinum oxides are still present after platinum dissolution, as determined by measuring the platinum oxide reduction peak at various intervals during potentiostatic hold

- An increase of the electrode potential leads to an increase in dissolution rate

- At $60{ }^{\circ} \mathrm{C}$, the dissolution is much slower than at $80^{\circ} \mathrm{C}$ 

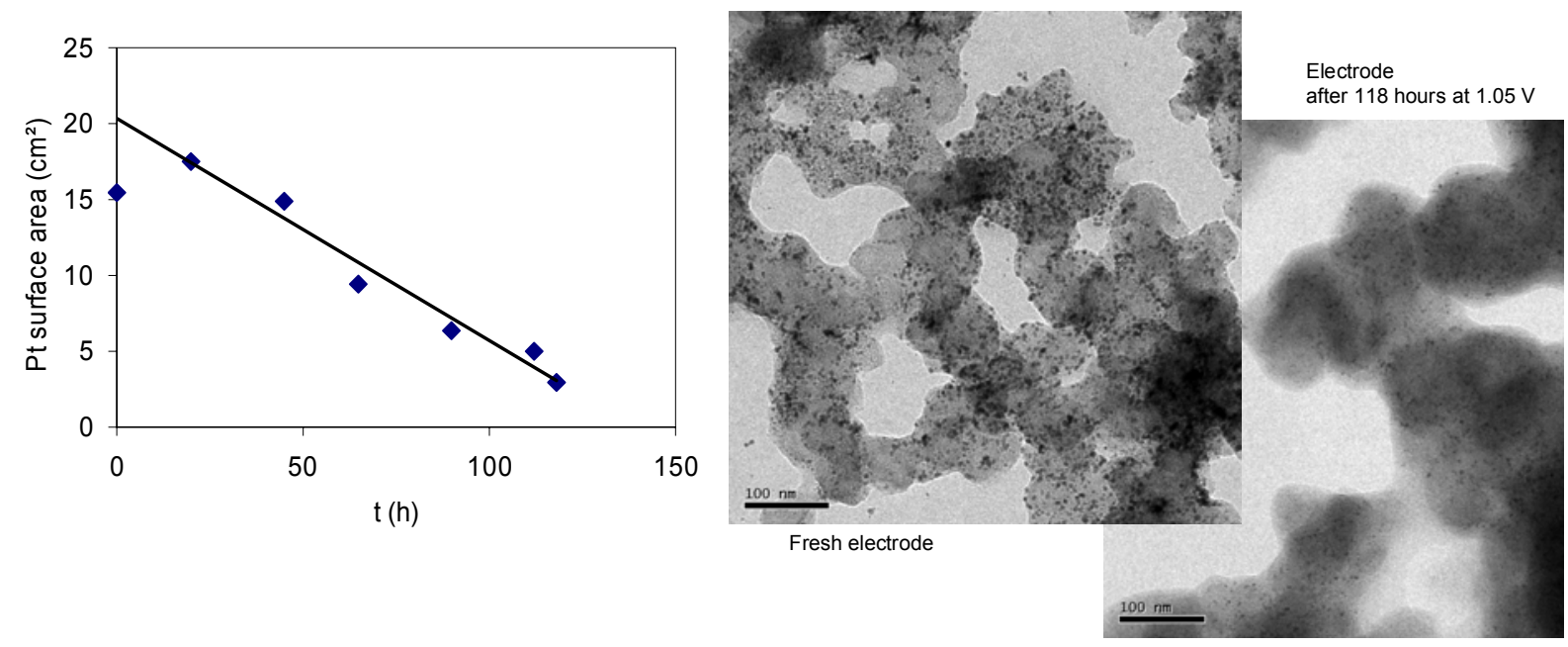

Figure 8. Left: Platinum surface area as measured by cyclic voltammetry versus time in $40 \mathrm{wt} \% \mathrm{Pt} / \mathrm{C}$, JM HiSpec ${ }^{\mathrm{TM}} 4000$, exposed to $1.05 \mathrm{~V}, 80^{\circ} \mathrm{C}$ in $1 \mathrm{M} \mathrm{HClO}_{4}$. Right: TEM pictures of same electrode, before potentiostatic hold and after 118 hours potentiostatic hold. TEM pictures previously published in Fuel Cells (10).

\section{Comparison of MEA voltage cycles and potentiostatic model studies}

The model studies on platinum, carbon and platinum/carbon catalysts have given insight in their stability over a wide range of conditions, which can be compared to the observations on full MEAs. As the maximum voltage applied at the MEA voltage cycling was $0.9 \mathrm{~V}$, continued carbon corrosion can be excluded, as the potentiostatic studies showed no substantial mass decrease of carbon under these conditions.

The model studies of platinum thin films have shown that at $0.9 \mathrm{~V}$ and $80{ }^{\circ} \mathrm{C}$, platinum dissolves. Although the studies at platinum/carbon catalysts were not performed at a potential as low as $0.9 \mathrm{~V}$, platinum dissolution from platinum supported on carbon is expected at $0.9 \mathrm{~V}$ and $80{ }^{\circ} \mathrm{C}$ as well, based on the solubility of bulk platinum under the same conditions and the extent of the dissolution measured for supported catalysts at 1.05 $\mathrm{V}$ and $80^{\circ} \mathrm{C}$.

The post test analysis of the MEAs exposed to voltage cycling, showed particle growth as well as platinum detected in the membrane, while the potentiostatic hold experiments in perchloric acid lead to platinum dissolution resulting in a decrease in platinum particle size. The presence of platinum in the membrane of the MEA proofs that platinum is indeed soluble at a cell voltage of $0.9 \mathrm{~V}$. As the electrolyte volume in the fuel cell electrode is small, and 30 seconds at $0.9 \mathrm{~V}$ are followed by 30 seconds at $0.7 \mathrm{~V}$, redeposition will take place to a large extent, leading to larger platinum particles following the Ostwald ripening mechanism. In the model studies, the electrolyte volume is much higher and the voltage is kept at a constant value, thus preventing redeposition. 


\section{Conclusions}

The stability of PEMFC MEAs and platinum/carbon electrodes has been proven ex situ and in situ to be insufficient under the conditions relevant for automotive operation. Voltage cycling and potentiostatic hold as well as freeze/thaw cycles lead to an unacceptable loss of platinum surface area. Changes in the electrodes have become the dominating factor in performance loss when chemically stable membranes are used in MEAs, provided these membranes are not too thin, as the MEA containing a $25 \mu \mathrm{m}$ membrane showed considerable thinning which was not observed in the MEA containing a $50 \mu \mathrm{m}$ membrane. The obtained results show that electrode materials presently used do not meet the requirements of automotive applications, and provide insight which operational conditions need to be avoided to extend the lifetime of PEMFCs.

\section{Acknowledgments}

The authors wish to thank E.F. Sitters and A.J. Grootjes (ECN) for performing MEA tests. This work was part of the Dutch EOS-LT PEMLIFE project, contract no.EOSLT01029, and EOS-LT Consortium PEMFC, contract nos. EOSLT 06005 and EOSLT 07005 supported by the Ministry of Economic Affairs.

\section{References}

1. F. A. de Bruijn, V. A. T. Dam and G. J. M. Janssen, Fuel Cells 8, 3 (2008).

2. G. J. M. Janssen, E. F. Sitters, and A. Pfrang, J.Power Sources, 191, 501 (2009)

3. E. Cho, J. J. Ko, H.Y. Ha, S. A. Hong, K. Y. Lee, T. W. Lim and I. H. Oh, J. Electrochem. Soc., 150, A1667 (2003).

4. E. Cho, J. J. Ko, H.Y. Ha, S. A. Hong, K. Y. Lee, T. W. Lim and I. H. Oh, J. Electrochem. Soc., 151, A661 (2004).

5. Q. Guo, Z. Qi, J. Power Sources, 160, 1269 (2006)

6. R. Mukundan, Y. S. Kim, F. Garzon, B. Pivovar, ECS Transactions, 1, 403 (2006)

7. N. Garland, T. Benjamin, and J. Kopasz, ECS Transactions, 11, 923 (2007).

8. S. J. C. Cleghorn, D. K. Mayfield, D. A. Moore, J. C. Moore, G. Rusch, T. W. Sherman, N. T. Sisofo, and U. Beuscher, J.Power Sources, 158, 446 (2006).

9. V. A. T. Dam and F. A. de Bruijn, J.Electrochem.Soc., 154, B494 (2007).

10. V.A.T. Dam, K. Jayasayee and F.A. de Bruijn, "Determination of the potentiostatic stability of PEMFC electro catalysts at elevated temperatures" accepted for publication in Fuel Cells: DOI: 10.1002/fuce.200800136. 